UPR-0867-T

FERMILAB-Pub-99/369-T

hep-ph/0001073

\title{
Alternative Supersymmetric Spectra
}

\author{
Lisa Everett*, Paul Langacker ${ }^{\dagger}$, Michael Plümacher ${ }^{\dagger}$, and Jing Wang ${ }^{\dagger}, * *$ \\ * Department of Physics \\ University of Michigan, Ann Arbor, MI 48109 , USA \\ $\dagger$ Department of Physics and Astronomy \\ University of Pennsylvania, Philadelphia PA 19104-6396, USA \\ ** Theoretical Physics Department \\ Fermi National Accelerator Laboratory \\ Batavia, Illinois, 60510, USA
}

(November 7, 2018)

\begin{abstract}
We describe the features of supersymmetric spectra, alternative to and qualitatively different from that of most versions of the MSSM. The spectra are motivated by extensions of the MSSM with an extra $U(1)^{\prime}$ gauge symmetry, expected in many grand unified and superstring models, which provide a plausible solution to the $\mu$ problem, both for models with supergravity and for gauge-mediated supersymmetry breaking. Typically, many or all of the squarks are rather heavy (larger than one TeV), especially for the first two families, as are the sleptons in the supergravity models. However, there is a richer spectrum of Higgs particles, neutralinos, and (possibly) charginos. Concrete examples of such spectra are presented, and the phenomenological implications are briefly discussed.
\end{abstract}




\section{Introduction.}

The minimal supersymmetric standard model (MSSM) and its simple extensions contain many free parameters associated with supersymmetry breaking. Most analyses have been based on two generic classes of models of soft supersymmetry breaking: (1) supergravity, in which supersymmetry breaking in a hidden sector is transmitted to the observable sector via supergravity. One usually assumes universality or at least a comparable scale for the soft parameters at the Planck scale. (2) Gauge-mediated models, with the breaking transmitted via messenger fields at relatively low energy, such as $10^{5} \mathrm{GeV}$. In both cases the scale of the soft supersymmetry breaking parameters ultimately sets the electroweak scale via radiative electroweak breaking, provided that the supersymmetric $\mu$ parameter is of a comparable magnitude. Once universality is relaxed there are many free parameters in supergravity, and there are many versions of gauge-mediation. However, in both cases a typical spectrum involves sparticle masses in the several hundred $\mathrm{GeV}$ range due to naturalness arguments; i.e., the mass scale of the superpartners should be in this range (and at most $\sim \mathcal{O}(1 \mathrm{TeV})$ ) for SUSY to explain the origin of the electroweak scale without excessive fine-tuning. Most studies of the implications for current and future colliders and precision measurements have been based on such a spectrum.

However, it is well known that naturalness does not necessarily require that all sparticles have masses below the $\mathrm{TeV}$ scale [1]. In the scalar sector, naturalness only constrains the masses of the third generation sfermions and the electroweak Higgs doublets, as these are the fields which have large Yukawa couplings and thus play dominant roles in radiative electroweak symmetry breaking. Therefore, the sparticle masses of the first and second generations can be significantly larger than the other sparticle (and particle) masses without violating naturalness criteria. Recent work has demonstrated that this hierarchy can be generated dynamically via renormalization group evolution (first pointed out in [2] and investigated in the context of grand unified models in [3]). In this scenario, the soft supersymmetry breaking scalar mass-squared parameters can be multi- TeV $(\sim 4 \mathrm{TeV})$ at the high scale (while the gaugino masses and scalar trilinear couplings are $\sim M_{W}$; the Higgs and third generation masses are driven to smaller values due to their large Yukawa couplings, while the first and second generations remain heavy. The results of a recent extension of this framework including the possibility of multi-TeV $A$ parameters 4 indicate that such inverted hierarchies can be generated with the first two generations up to $\sim 20 \mathrm{TeV}$. This scenario has distinctive implications (such as in collider searches for superpartners; see, e.g., [2.5]), and can be advantageous phenomenologically, as stringent laboratory constraints on the SUSY parameter space from flavor-changing neutral currents (FCNC) and CP-violation can be considerably weakened [回]. Another possibility in supergravity models pointed out in [6] is that since the Higgs soft mass-squared parameter at the electroweak scale can be

quite insensitive to the initial values of the scalar masses due to "focus-point" behavior of the RGE's, scalar masses for all three generations of squarks and sleptons of order $2-3 \mathrm{TeV}$ can be consistent with naturalness (see [7] for a discussion in the context of gauge-mediated models).

The purpose of this paper is to point out that there is another class of (string-motivated) models based on gauge extensions of the MSSM with an additional $U(1)^{\prime}$ gauge group in which this type of spectrum is naturally achieved. In these models, it has been shown that the $U(1)^{\prime}$ may be broken at the $\mathrm{TeV}$ scale by a radiative mechanism analogous to 
that for electroweak breaking provided there are sufficiently large Yukawa couplings of a standard model singlet $S$ which carries $U(1)^{\prime}$ charge [8]. Such extended gauge groups, exotic particle content, and large Yukawa couplings are generically present in classes of quasirealistic perturbative superstring constructions. These models also provide an alternative resolution to the $\mu$ problem of the MSSM, since gauge invariance can forbid the elementary $\mu$ term while an effective $\mu$ term can be generated via a trilinear coupling of the SM singlet to the two electroweak Higgs doublets [8.9]. Furthermore, the enhanced symmetry avoids the problems of domain walls, which are common to models involving an effective $\mu$ generated by the VEV of a scalar but not associated with the breaking of an extra gauge symmetry [10].

In this framework the VEV of the singlet field sets the scale of the $Z^{\prime}$ mass. This VEV is generally of order several $\mathrm{TeV}$ since the nonobservation of an additional $Z^{\prime}$ boson and the stringent constraints on the $Z-Z^{\prime}$ mixing angle $\alpha_{Z-Z^{\prime}}$ typically require that the mass of the $Z^{\prime}$ is significantly heavier than the $Z$ mass (the lower bounds on $M_{Z^{\prime}}$ are model dependent, but are in the range of $500 \mathrm{GeV}$ to $1 \mathrm{TeV}$ or so). Since the singlet VEV is achieved radiatively, its value generally sets the scale of the required initial values of the soft breaking parameters. Typical supersymmetry breaking parameters are at the TeV scale, at least for the first two generations. However, there is typically a much richer spectrum of Higgs particles and neutralinos, as well as the $Z^{\prime}$ and (usually) exotic fermions and their partners. Specific models based on perturbative heterotic string constructions also involve extended chargino sectors [11].

Since the electroweak and $U(1)^{\prime}$ symmetry breaking are coupled in these models, the large ratio of the $Z^{\prime}$ and $Z$ masses requires a certain amount of tuning of the parameters (cancellations are needed for the expectation values of the Higgs doublets to be sufficiently small). Nevertheless, such models are worth exploring as viable alternatives to the MSSM which are well motivated theoretically both within quasi-realistic string constructions and GUT models. An additional motivation to consider such models seriously arises from recent precision electroweak data. The $Z$ lineshape and atomic parity data hint at the existence

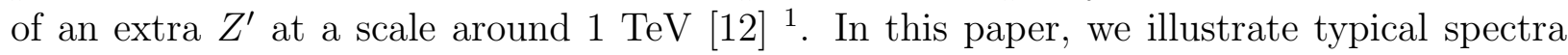
from several concrete models, some with supergravity-mediated SUSY soft breaking parameters and another with gauge-mediated supersymmetry breaking, and comment briefly on phenomenological implications.

Results: Alternative Supersymmetric Spectra.

The models we consider are extensions of the MSSM with an additional nonanomalous $U(1)^{\prime}$ gauge symmetry and additional matter fields, typically including both SM singlets (with $U(1)^{\prime}$ charges) and SM exotics. Such models are motivated from a class of quasirealistic (perturbative heterotic) superstring models [11]. It was shown in [11] that after vacuum restabilization this class of string models generically contain extended Abelian gauge structures and additional matter content at the string scale. The trilinear couplings, which

\footnotetext{
${ }^{1}$ The implications of the atomic parity data alone have been considered recently in [13]. For earlier references, see [12].
} 
can be calculated exactly in string perturbation theory, usually include the top quark Yukawa coupling and the coupling between Higgs doublets and a singlet field, an effective $\mu$-term. The coefficients of these couplings are calculable in string theory, and are of $\mathcal{O}(1)$. A phenomenological analysis of an explicit four-dimensional string model with these features [11] demonstrated that the large Yukawa couplings trigger the radiative breaking of the $U(1)^{\prime}$ by driving the soft supersymmetry breaking mass-squared parameters of the SM singlet fields $S_{i}$ negative at low energies via renormalization group evolution, as argued on general grounds in 8, 14, 15. For supergravity models with such large Yukawa couplings the $U(1)^{\prime}$ breaking will either be at the electroweak scale (i.e., up to a $\mathrm{TeV}$ or so) [14 or at a large intermediate scale [16] if the breaking occurs along a $D$-flat direction (we do not consider the intermediate scale $Z^{\prime}$ case further in this paper). Electroweak scale breaking can also be implemented in models with gauge-mediated supersymmetry breaking [17, 18.

A general analysis of these scenarios in the supergravity-mediated supersymmetry breaking framework was analyzed in a minimal model with no additional exotics [14]. However, this model is not $U(1)^{\prime}$ anomaly free, and thus does not have the necessary ingredients for a fully realistic theory, and thus the case of a string-motivated and anomaly-free $E_{6}$-type model was proposed in [14] and studied in [15]. These analyses demonstrated that there are corners of parameter space for which a phenomenologically acceptable $Z-Z^{\prime}$ hierarchy at the electroweak scale can be obtained. In these scenarios, the $U(1)^{\prime}$ breaking is radiative and triggered by a large $\mathcal{O}(\mathrm{TeV}) \mathrm{SM}$-singlet $\mathrm{VEV}$. This solution provides a $Z^{\prime}$ mass close to the natural upper limit of $1-2 \mathrm{TeV}$, with the electroweak scale achieved via cancellations that require a certain amount of tuning of the soft mass parameters. This is the least desirable feature of these models.

In all these models, the low energy spectra displayed features that are different from the standard MSSM spectrum. In general, the requirement of a phenomenologically acceptable $Z-Z^{\prime}$ hierarchy leads to low energy values of some or all of the scalar masses that are generically a few $\mathrm{TeV}$. This feature can be understood heuristically within the supergravity-mediated supersymmetry breaking scenarios, where the boundary conditions are implemented at the string scale $\sim M_{G}$ [ as follows. In the limit of $\langle S\rangle \gg\left\langle H_{1}\right\rangle,\left\langle H_{2}\right\rangle$, the $Z^{\prime}$ mass is set by the soft mass-squared parameter $m_{S}^{2}$ of the singlet $S$ at low energies by: $M_{Z^{\prime}} \sim \sqrt{-2 m_{S}^{2}}$ (in this limit the $U(1)^{\prime}$ breaking can be considered separately from the electroweak breaking). To obtain the large and negative $m_{S}^{2}$ parameter at low energies and to avoid large fine-tuning, in general it is necessary that the singlet couples with $\mathcal{O}(1)$ Yukawa couplings to additional SM exotic quarks, as such couplings drive the singlet masssquared parameter strongly to negative values. In this case, the RG evolution provides the

\footnotetext{
${ }^{2}$ Another possibility is that the symmetry breaking is driven by a large value of the soft supersymmetry breaking trilinear coupling [14]. However, this solution yields a light $Z^{\prime}$ that is phenomenologically excluded except in the case of models with certain (leptophobic) couplings, and thus we do not consider this scenario further in this paper.

${ }^{3}$ The small discrepancy between the observed unification scale $M_{G}$ and the perturbative heterotic string scale is not significant for the cases considered here.
} 
desired value of $m_{S}^{2}$ at low energies provided that the scale of the soft mass-squares of the exotic quarks at $M_{G}$ is about a few TeV. This scale determines the typical magnitudes of the soft mass-squared parameters in such phenomenologically acceptable models (which are not excessively tuned either at the electroweak scale or at the high scale). The first and second generation sparticles (whose soft mass-squared parameters do not run significantly due to their smaller Yukawa couplings) are typically a few TeV. However, a certain amount of fine-tuning is needed to obtain low energy values of the Higgs soft mass-squared parameters of the order of the electroweak scale. Since the $U(1)^{\prime}$ symmetry breaking is at the TeV scale, there are also additional Higgs bosons and neutralinos in the low energy spectrum. In the large $\langle S\rangle$ limit, some of these additional states acquire masses $\sim M_{Z^{\prime}}$. We note that in the explicit string-derived model analyzed in [11], the couplings are more complicated, and an extended Higgs sector must be invoked to achieve a realistic $Z-Z^{\prime}$ hierarchy. As a result, the low energy spectrum includes additional charginos as well as Higgs bosons and neutralinos.

To illustrate these features, we now turn to several supergravity models [14,15] and demonstrate the symmetry breaking pattern and the low energy spectrum explicitly. For the sake of simplicity, the models discussed are those with a minimal Higgs sector of two electroweak doublets and one SM singlet. For each model, we display the relevant mass parameters at both the electroweak scale and the string (or GUT) scale in Table I and II, and present the detailed low energy spectra explicitly in Table III.

The first example we consider of an anomaly-free model with $U(1)^{\prime}$ charges that allow an induced $\mu$ term $\left(Q_{1}+Q_{2}+Q_{S}=0\right)$, where $Q_{1}, Q_{2}$, and $Q_{S}$ are respectively the $U(1)^{\prime}$ charges of $H_{1}, H_{2}$, and $S$, and also does not include additional SM exotics was first presented in the Appendix of [14]. The charge assignments in this model (in self-evident notation) are given by

$$
\begin{array}{ll}
Q_{E_{3}}=Q_{2}-Q_{1}, & Q_{L_{3}}=-Q_{2}, \\
Q_{Q_{3}}=-\frac{1}{3} Q_{1}, & \\
Q_{S}=-\left(Q_{1}+Q_{2}\right), \\
Q_{D_{3}}=\frac{1}{3}\left(Q_{1}+3 Q_{2}\right), & Q_{U_{3}}=\frac{1}{3}\left(Q_{1}-3 Q_{2}\right),
\end{array}
$$

for arbitrary $Q_{1}$ and $Q_{2}$, and the first and second families have zero $U(1)^{\prime}$ charges. We stress that this model is only semi-realistic; while these charge assignments are consistent with gauge invariance conditions for the top quark $Q_{U_{3}}+Q_{Q_{3}}+Q_{2}=0$ and the tau lepton $Q_{E 3}+Q_{L 3}+Q_{1}=0$ Yukawa interactions, the bottom quark Yukawa interaction (and those of the first two generations) is forbidden by the symmetry. The bottom quark mass can be generated from a higher-dimensional operator, but its value is suppressed by the $U(1)^{\prime}$ breaking scale and thus is too small. However, we present this model as a minimal example in which to display the patterns of the $U(1)^{\prime}$ symmetry breaking and resulting spectra. Nonuniversal boundary conditions (or the addition of exotics) at the string scale are required to drive the singlet mass-squared parameter negative at the electroweak scale in this model. The boundary conditions at the string scale are presented in Table I, for an example in which $M_{Z^{\prime}}=1 \mathrm{TeV}$, the $Z-Z^{\prime}$ mixing is $\alpha_{Z-Z^{\prime}}=2 \times 10^{-3}$, and $\tan \beta=2$.

Another example, which provides acceptable anomaly free $U(1)^{\prime}$ quantum numbers and is approximately consistent with gauge unification, is a string-motivated model with $E_{6}$ particle content (without $E_{6}$-type relations among the Yukawa couplings) [14, 15]. The particle 
content of the model under consideration includes three $E_{6} 27$-plets, each of which includes an ordinary family, two Higgs-type doublets, two standard model singlets, and two exotic $S U(2)$-singlet quarks with charge $\pm 1 / 3$. We also assume a single vector-like pair of Higgstype doublets from a $27+27^{*}$, which does not introduce any anomalies. The particle content is consistent with gauge unification. It is further assumed that only a subset of these fields (the SM Higgs doublets, SM singlet $S$, and an exotic quark pair $D$ and $\bar{D}$ ) play significant roles in the radiative breaking due to the presence of trilinear superpotential couplings (with $\mathcal{O}(1)$ coefficients) of the form $S H_{1} \cdot H_{2}$ and $S D \bar{D}$.

The $U(1)^{\prime}$ symmetry breaking patterns of this model were analyzed in detail in [15] assuming a general set of supergravity-mediated soft supersymmetry breaking mass parameters; we refer the reader to this work for further details. In general, non-universal boundary conditions are required to achieve the desired hierarchy. The soft parameters and low energy spectrum of the first numerical example of this model $\left(E_{6}(\mathrm{UA})\right)$, which includes only the dominant effect of the top Yukawa couplings and assumes universal $A$-parameters at $M_{G}$, are presented for a case in which $M_{Z^{\prime}}=1700 \mathrm{GeV}, \alpha_{Z-Z^{\prime}}=2 \times 10^{-3}$. The second numerical example of this model $\left(E_{6}(\mathrm{NUA})\right)$, which has non-universal $A$-terms and also includes the bottom, tau and charm Yukawas, is presented for a case in which $M_{Z^{\prime}}=1600 \mathrm{GeV}$, $\alpha_{Z-Z^{\prime}}=1 \times 10^{-3}$. The non-universal $A$ parameters result in a different pattern in the squark spectrum, as presented in Table III.

This class of models was also analyzed recently assuming gauge-mediated supersymmetry breaking [17]. The particle content of the observable sector in the particular example considered includes the MSSM fields, as well as vector pair of quark singlets $(D$ and $\bar{D})$, and an additional singlet field whose couplings to the two Higgs doublets and to $D, \bar{D}$ are allowed by gauge invariance. The supersymmetry breaking scale is set to the standard value of $10^{5} \mathrm{GeV}$. In this model it is assumed that the messenger fields are not charged under the $U(1)^{\prime}$ symmetry; therefore, the soft mass-square of the singlet field is zero at the messenger scale. As a result, to achieve a desired value of $m_{S}^{2}$ at the electroweak scale over the short period of RGE running (from $10^{5} \mathrm{GeV}$ to $\sim 10^{2} \mathrm{GeV}$ ), the soft mass-squares of the scalar exotic quarks generated at the messenger scale are required to be of order $\mathrm{TeV}$, which thus sets the mass scale for the masses of the other squarks. A numerical example of the model is presented in Table I and II, with the $Z^{\prime}$ mass of $1110 \mathrm{GeV}$ and the mixing angle $\alpha_{Z-Z^{\prime}}=0.004$.

An inspection of Table III indicates that the low energy spectra of each of these models share several common features. The scalar particles generally have masses at the $\mathrm{TeV}$ scale, higher than the standard scenarios in the MSSM. In particular, the squarks of the first and second generation have masses about $1-3 \mathrm{TeV}$ in each of the examples. The first $E_{6}$ example, with universal $A$-parameters at the GUT scale, predicts a hierarchy between the third family and the first two family squarks; in particular the stops and sbottoms are much lighter. In contrast, in the second $E_{6}$ example with non-universal $A$-parameters, the squarks of the three families can have masses of the same order. In the case in which the third family sparticles are also heavy, there is an additional tuning issue because the stops enter at the loop level of the Higgs potential; however, given that cancellations between terms of order $(\mathrm{TeV})^{2}$ already are present at the tree level, the tuning is not significantly worsened for stops with $\mathrm{TeV}$ masses. In the gauge-mediated model, the squarks (including the exotics) acquire $\mathrm{TeV}$ scale masses at the messenger scale; since the running time is very short (from 
$10^{5} \mathrm{GeV}$ to $\left.\sim 10^{2} \mathrm{GeV}\right)$, their masses stay heavy $\mathcal{O}(\mathrm{TeV})$. In the slepton sector, both of the $E_{6}$ models predict heavy sleptons with masses above around one $\mathrm{TeV}$, while the gauge mediated model has sleptons of a few hundred $\mathrm{GeV}$ due to the gauge coupling hierarchy at the messenger scale (see also [7]).

We now turn to a discussion of the Higgs and neutralino sectors, as discussed in detail in [14. With the assumption of a minimal set of Higgs fields, in addition to the MSSM Higgs bosons there is one additional neutral CP-even Higgs boson which is predominantly the real component of the singlet field and has a mass $\sim M_{Z^{\prime}}$ in the large $\langle S\rangle$ limit. In the neutralino sector, there are two additional neutralinos: one extra gaugino (corresponding to the fermionic partner of the $Z^{\prime}$ ) and an extra Higgsino (corresponding to the fermionic partner of the $S$ field). In the large $\langle S\rangle$ limit, these neutralinos mix and have masses controlled by $M_{Z^{\prime}}$. We also note that the upper bound on the tree level mass of the lightest Higgs receives a contribution from the $U(1)^{\prime} D$-term [14] and thus can be heavier than that of the MSSM, which is a particular feature of this class of models.

\section{Concluding Remarks.}

The purpose of this paper has been to emphasize two main points: (i) supersymmetric models with an additional $U(1)^{\prime}$ gauge symmetry broken at the TeV scale are well motivated extensions of the MSSM both theoretically and phenomenologically, and (ii) the characteristic low energy mass spectra of this class of models exhibit patterns which have distinctive features compared to that of the MSSM. In particular, the strong phenomenological constraints on the $Z^{\prime}$ mass and mixing with the ordinary $Z$ dictate that the $U(1)^{\prime}$ is broken by a large singlet VEV of order several $\mathrm{TeV}$, which sets the initial scale of the soft scalar mass-squared parameters. The resulting low energy spectra generically have heavy scalars, as well as a richer spectrum of Higgs bosons, neutralinos, and possibly charginos. In this scenario, the electroweak scale is generated by cancellations, which in turn suggests a natural upper limit of the mass scale of the heavy scalars (and the $Z^{\prime}$ mass) to be of order several $\mathrm{TeV}$ to avoid excessive fine-tuning.

We conclude with a brief discussion of the phenomenological implications of the mass spectra in this class of models. In general, the heavy squarks (and sleptons in the supergravity models) can lead to distinctive phenomenological signatures and can ease the strong constraints on the SUSY parameter space from FCNC and CP violation (see e.g. 19,20,21 and references therein for the analysis of these processes within the MSSM) as discussed recently in [1,5]. In the models considered here the heavy scalars in these models are typically in the range $1-3 \mathrm{TeV}$; as such, it is well known that flavor changing neutral current (FCNC) operators due to box diagrams are typically suppressed compared to the MSSM because of the larger scale, although one must still rely somewhat on the assumption that the soft scalar mass-squares that are generated due to some (unknown) supersymmetry breaking mechanism are diagonal in flavor space. In our RGE analysis, the scalar mass-squares and the $A$ terms are assumed to be diagonal (but not necessarily universal). For this reason, we will not go into a detailed analysis of the implications of the spectra presented in our paper on the FCNC and CP-violating processes. Instead, note that with squark masses of order a few $\mathrm{TeV}$, the requirement of universality of the soft scalar masses can be relaxed compared to the case of the MSSM. Namely, the splitting between the scalar masses of the three quark families $\left|m_{q_{1}}-m_{q_{2}}\right| / m_{q_{3}}$ can be as large as $\mathcal{O}(1)$, while still satisfying present 
experimental bounds on FCNC. We also point out that in the models considered there can also be new flavor changing effects due to family non-universal $U(1)^{\prime}$ couplings (e.g., the string-derived model in [11] has family non-universal $U(1)^{\prime}$ charges). While such family non-universal couplings are subject to severe constraints for the couplings of the first two generations, the third generation couplings are less constrained. An complete analysis of these effects is currently underway [22].

In addition to the effects from the heavy scalars, the extended gauge, Higgs, neutralino, and (possibly) chargino sectors implicit in these models have a number of phenomenological consequences. The implications include new expectations for precision experiments [12] and collider searches, and possibly new patterns for dark matter [23. In addition, the presence of the $U(1)^{\prime}$ symmetry and the exotics can have effects on $R$-parity violation, neutrino masses, quark and charged lepton masses and mixings, and scenarios for baryogenesis. A

comprehensive study of such issues is beyond the scope of this paper and is deferred for a future study.

\section{ACKNOWLEDGMENTS}

We thank M. Cvetič, J. Erler, and J. R. Espinosa for helpful discussions and suggestions. This work is supported in part by the U.S. Department of Energy Grants No. EY-76-02-3071 (P.L.,M.P.), DE-FG02-95ER40899 (L.E.), and DE-AC02-76CH03000 (J.W.), and in part by

the Feodor Lynen Program of the Alexander von Humboldt Foundation (M.P.). 


\section{REFERENCES}

[1] M. Drees, Phys. Rev. D33,1468 (1986); S. Dimopoulos and G.F. Giudice, Phys. Lett. B357, 573 (1995); A. Pomarol and D. Tommasini, Nucl. Phys. B466, 3 (1996); A.G. Cohen, D.B. Kaplan and A.E. Nelson, Phys. Lett. B388, 588 (1996); K. Agashe and M. Graesser, Phys. Rev. D59, 015007 (1999); D. Wright, hep-ph/9801449; G. Dvali and A. Pomarol, Phys. Rev. Lett. 77, 3728 (1996); ibid., Nucl. Phys. B522, 3 (1998); R.N. Mohapatra and A. Riotto, Phys. Rev. D55, 1 (1997); R.-J. Zhang, Phys. Lett. B402, 101 (1997); A.E. Nelson and D. Wright, Phys. Rev. D56, 1598 (1997); J. Hisano, K. Kurosawa and Y. Nomura, Phys. lett. B445, 316 (1999); H.P. Nilles and N. Polonsky, Phys. Lett. B412, 69 (1997); D.E. Kaplan, F. Lepeintre, A. Masiero, A.E. Nelson and A. Riotto, hep-ph/9806430.

[2] J.L. Feng, C. Kolda and N. Polonsky, Nucl. Phys. B546, 3 (1999).

[3] J. Bagger, J.L. Feng and N. Polonsky, hep-ph/9905292.

[4] J. Bagger, J.L. Feng and N. Polonsky and R.-J. Zhang hep-ph/9911255.

[5] V. Barger, C. Kao and R.-J. Zhang hep-ph/9911510.

[6] J. L. Feng, K. T. Matchev and T. Moroi, hep-ph/9908309, hep-ph/9909334.

[7] K. Agashe, hep-ph/9910497.

[8] M. Cvetič and P. Langacker, Phys. Rev. D 54, 3570 (1996) and Mod. Phys. Lett. A 11, 1247 (1996).

[9] D. Suematsu and Y. Yamagishi, Int. J. Mod. Phys. A 10, 4521 (1995).

[10] S. F. King and P. L. White, Phys. Rev. D52, 4183 (1995), and references therein.

[11] Examples are studied in detail in G. Cleaver, M. Cvetič, J. R. Espinosa, L. Everett, P. Langacker, and J. Wang, Phys. Rev. D 59, 055005 (1999); ibid. 115003 (1999).

[12] J. Erler and P. Langacker, hep-ph/9910315.

[13] R. Casalbuoni et al., Phys. Lett. 460B, 135 (1999); J.L. Rosner, Phys. Rev. D61, 016006 (2000).

[14] M. Cvetič, D. A. Demir, J. R. Espinosa, L. Everett and P. Langacker, Phys. Rev. D 56, 2861 (1997), Phys. Rev. D 58, 119905 (1998) (E).

[15] P. Langacker and J. Wang, Phys. Rev. D 58, 115010 (1998).

[16] G. Cleaver, M. Cvetič, J.R. Espinosa, L. Everett and P. Langacker, Phys. Rev. D57, 2701 (1998).

[17] P. Langacker, N. Polonsky and J. Wang, Phys. Rev. D60, 115005 (1999).

[18] H. Cheng, B. A. Dobrescu and K. T. Matchev, Phys. Lett. B 439, 301 (1998); Nucl. Phys. B 543, 47 (1999).

[19] F. Gabbiani, E. Gabrielli, A. Masiero and L. Silvestrini, Nucl. Phys. B 477, 321 (1996).

[20] M. Misiak, S. Pokorski and J. Rosiek, Supersymmetry and FCNC effects, in Heavy Flavours II (World Scientific Publishing Co., Singapore), eds. A.J. Buras and M. Lindner, p. 795.

[21] Y. Grossman, Y. Nir and R. Rattazzi, CP violation beyond the standard model, in Heavy Flavours II (World Scientific Publishing Co., Singapore), eds. A.J. Buras and M. Lindner, p. 755.

[22] P. Langacker and M. Plümacher, work in progress.

[23] B. de Carlos and J. R. Espinosa, Phys. Lett. B 407, 12 (1997). 


\begin{tabular}{|c|c|c|c|c|c|c|c|c|}
\hline & \multicolumn{2}{|c|}{$\mathrm{SF}$} & \multicolumn{2}{|c|}{$E_{6}(\mathrm{UA})$} & \multicolumn{2}{|c|}{$E_{6}(\mathrm{NUA})$} & \multicolumn{2}{|c|}{ GMSB } \\
\hline$h_{t}$ & 0.72 & 1.02 & 1.7 & 1.27 & 0.85 & 1.26 & 0.84 & 0.98 \\
\hline$h_{S}$ & 0.72 & 0.57 & 1.7 & 0.21 & 0.85 & 0.24 & 0.47 & 0.40 \\
\hline$h_{D}$ & - & - & 1.7 & 1.27 & 0.85 & 1.23 & 0.70 & 0.84 \\
\hline$h_{b}$ & - & - & - & - & 0.01 & 0.06 & 0.30 & 0.42 \\
\hline$h_{c}$ & - & - & - & - & $3 \times 10^{-3}$ & 0.01 & - & - \\
\hline$h_{\tau}$ & - & - & - & - & 0.01 & 0.02 & 0.17 & 0.18 \\
\hline$M_{1}$ & 100 & 41 & 371 & 54 & 650 & 95 & 147 & 135 \\
\hline$M_{2}$ & 100 & 82 & 371 & 108 & 650 & 190 & 279 & 269 \\
\hline$M_{3}$ & 100 & 290 & 371 & 371 & 650 & 650 & 801 & 955 \\
\hline$M_{1}^{\prime}$ & 100 & 35 & 371 & 52 & 650 & 92 & 0 & 0 \\
\hline$A_{Q}$ & 1800 & 440 & -2960 & 281 & -2180 & 494 & 0 & 449 \\
\hline$A_{S}$ & 2240 & 600 & -2960 & -188 & 2180 & 50 & 0 & -53 \\
\hline$A_{D}$ & - & - & -2960 & 284 & 2180 & 801 & 0 & 494 \\
\hline$A_{b}$ & - & - & -2960 & -1550 & -2180 & 142 & 0 & 574 \\
\hline$A_{c}$ & - & - & -2960 & -1520 & -2180 & 316 & - & - \\
\hline$A_{\tau}$ & - & - & -2960 & -2700 & 2180 & 2410 & 0 & 24 \\
\hline
\end{tabular}

TABLE I. The Yukawa couplings $h_{i}$, gaugino masses $M_{a}$, and the soft trilinear couplings $A_{i}$ for each discussed model. SF refers to the model in eq. (11) with $Q_{1}=-1, Q_{2}=-1 / 2$. $E_{6}$ (UA) and $E_{6}$ (NUA) are respectively the models with universal and non-universal $A$ terms. GMSB is the $E_{6}$ model with gauge mediated supersymmetry breaking. $h_{t, b, c, \tau}$ are the Yukawa couplings for the top, bottom, charm, and tau, respectively. $h_{S}$ is the Yukawa coupling of the singlet $S$ to the Higgs doublets, and $h_{D}$ (for the $E_{6}$ models) is the Yukawa coupling of $S$ to the exotic quark pair $D, \bar{D}$. For each model the first column refers to the parameters at the string or messenger scale, and the second at the electroweak scale. For each table, the blank entries refer to arbitrary quantities that are essentially irrelevant to the symmetry breaking pattern, while the dashes refer to small quantities that are neglected or to states that are absent in the model. 


\begin{tabular}{|c|c|c|c|c|c|c|c|c|}
\hline & \multicolumn{2}{|c|}{$\mathrm{SF}$} & \multicolumn{2}{|c|}{$E_{6}(\mathrm{UA})$} & \multicolumn{2}{|c|}{$E_{6}(\mathrm{NUA})$} & \multicolumn{2}{|c|}{ GMSB } \\
\hline$m_{1}^{2}$ & $(1150)^{2}$ & $(577)^{2}$ & $(1920)^{2}$ & $(302)^{2}$ & $(1660)^{2}$ & $-(721)^{2}$ & $\overline{(351)^{2}}$ & $(83)^{2}$ \\
\hline$m_{2}^{2}$ & $(2660)^{2}$ & $-(302)^{2}$ & $(5550)^{2}$ & $(681)^{2}$ & $(5940)^{2}$ & $(442)^{2}$ & $(351)^{2}$ & $-(778)^{2}$ \\
\hline$m_{S}^{2}$ & $(1220)^{2}$ & $-(707)^{2}$ & $(3600)^{2}$ & $-(1210)^{2}$ & $(3830)^{2}$ & $-(1150)^{2}$ & 0 & $-(821)^{2}$ \\
\hline$m_{Q_{3}}^{2}$ & $(2730)^{2}$ & $(1000)^{2}$ & $(2920)^{2}$ & $(131)^{2}$ & $(3170)^{2}$ & $(1000)^{2}$ & $(1350)^{2}$ & $(1410)^{2}$ \\
\hline$m_{u_{3}}^{2}$ & $(2250)^{2}$ & $(1000)^{2}$ & $(4180)^{2}$ & $(181)^{2}$ & $(4550)^{2}$ & $(1010)^{2}$ & $(1310)^{2}$ & $(1290)^{2}$ \\
\hline$m_{D}^{2}$ & - & - & $(1630)^{2}$ & $(993)^{2}$ & $(1630)^{2}$ & $(1000)^{2}$ & $(1310)^{2}$ & $(1440)^{2}$ \\
\hline$m_{\bar{D}}^{2}$ & - & - & $(1600)^{2}$ & $(871)^{2}$ & $(1650)^{2}$ & $(1000)^{2}$ & $(1309)^{2}$ & $(1440)^{2}$ \\
\hline$m_{L_{3}}^{2}$ & & & $(2470)^{2}$ & $(2520)^{2}$ & $(1000)^{2}$ & $(1100)^{2}$ & $(351)^{2}$ & $(363)^{2}$ \\
\hline$m_{L_{1,2}}^{2}$ & & & $(2470)^{2}$ & $(2520)^{2}$ & $(1000)^{2}$ & $(1100)^{2}$ & $(351)^{2}$ & $(364)^{2}$ \\
\hline$m_{E_{3}}^{2}$ & & & $(2470)^{2}$ & $(2387)^{2}$ & $(1000)^{2}$ & $(977)^{2}$ & $(161)^{2}$ & $(160)^{2}$ \\
\hline$m_{E_{1,2}}^{2}$ & & & $(2470)^{2}$ & $(2387)^{2}$ & $(1000)^{2}$ & $(977)^{2}$ & $(161)^{2}$ & $(164)^{2}$ \\
\hline$m_{N}^{2}$ & - & - & $(2470)^{2}$ & $(2500)^{2}$ & $(1000)^{2}$ & $(1040)^{2}$ & & \\
\hline$m_{Q_{1,2}}^{2}$ & & & $(2470)^{2}$ & $(2560)^{2}$ & $(1000)^{2}$ & $(1590)^{2}$ & $(1350)^{2}$ & $(1510)^{2}$ \\
\hline$m_{u_{1,2}}^{2}$ & & & $(2470)^{2}$ & $(2630)^{2}$ & $(1000)^{2}$ & $(1580)^{2}$ & $(1310)^{2}$ & $(1470)^{2}$ \\
\hline$m_{d_{3}}^{2}$ & & & $(2470)^{2}$ & $(2520)^{2}$ & $(1000)^{2}$ & $(1520)^{2}$ & $(1310)^{2}$ & $(1440)^{2}$ \\
\hline$m_{d_{1,2}}^{2}$ & & & $(2470)^{2}$ & $(2520)^{2}$ & $(1000)^{2}$ & $(1520)^{2}$ & $(1310)^{2}$ & $(1470)^{2}$ \\
\hline$m_{H_{u}}^{2}$ & - & - & $(2470)^{2}$ & $(2410)^{2}$ & $(1000)^{2}$ & $(1040)^{2}$ & & \\
\hline$m_{H_{d}}^{2}$ & - & - & $(2470)^{2}$ & $(2522)^{2}$ & $(1000)^{2}$ & $(1100)^{2}$ & & \\
\hline$m_{H_{3}}^{2}$ & - & - & $(2470)^{2}$ & $(2560)^{2}$ & $(1000)^{2}$ & $(11300)^{2}$ & & \\
\hline$m_{S_{1,2}}^{2}$ & - & - & $(2470)^{2}$ & $(2500)^{2}$ & $(1000)^{2}$ & $(1040)^{2}$ & & \\
\hline$m_{D_{1,2}}^{2}$ & - & - & $(2470)^{2}$ & $(2570)^{2}$ & $(1000)^{2}$ & $(1550)^{2}$ & & \\
\hline$m_{\bar{D}_{1,2}}^{2} \mid$ & - & - & $(2470)^{2}$ & $(2520)^{2}$ & $(1000)^{2}$ & $(1520)^{2}$ & & \\
\hline
\end{tabular}

TABLE II. The soft SUSY breaking mass-squared parameters for the models discussed. $m_{1,2}^{2}$ and $m_{Q_{i}}^{2}, m_{u_{i}}^{2}, m_{d_{i}}^{2}, m_{L_{i}}^{2}, m_{E_{i}}^{2}$ are the mass parameters of the MSSM Higgs doublets and the MSSM matter fields, while $m_{S}^{2}$ is the mass parameter of the SM singlet $S$. In the $E_{6}$ models there are also exotic quarks $D, \bar{D}$, additional doublets $H_{u, d, 3}$, and singlets $S_{1,2}, N$, with soft mass-squares given above (in self-evident notation). 


\begin{tabular}{|c|c|c|c|c|}
\hline & $\mathrm{SF}$ & $E_{6}(\mathrm{UA})$ & $E_{6}(\mathrm{NUA})$ & GMSB \\
\hline$M_{Z^{\prime}}$ & 1000 & 1720 & 1620 & 1120 \\
\hline$\alpha_{Z-Z^{\prime}}$ & $2 \times 10^{-3}$ & $2 \times 10^{-3}$ & $10^{-3}$ & $4 \times 10^{-3}$ \\
\hline$m_{h_{1}^{0}}$ & 115 & 62 & 79 & 125 \\
\hline$m_{h_{2}^{0}}$ & 657 & 772 & 348 & 998 \\
\hline$m_{h_{3}^{0}}$ & 1000 & 1720 & 1620 & 1090 \\
\hline$m_{A^{0}}$ & 662 & 773 & 342 & 993 \\
\hline$m_{H^{ \pm}}$ & 657 & 774 & 349 & 994 \\
\hline$m_{\tilde{\chi}_{1}^{0}}$ & 719 & -1690 & -1580 & -1120 \\
\hline$m_{\tilde{\chi}_{2}^{0}}$ & 684 & -870 & -939 & -1060 \\
\hline$m_{\tilde{\chi}_{3}^{0}}$ & 592 & 53 & 94 & 142 \\
\hline$m_{\tilde{\chi}_{4}^{0}}$ & 578 & 103 & 183 & 266 \\
\hline$m_{\tilde{\chi}_{5}^{0}}$ & 72 & 876 & 946 & 1060 \\
\hline$m_{\tilde{\chi}_{6}^{0}}$ & 37 & 1745 & 1671 & 1120 \\
\hline$m_{\tilde{\chi}_{1}^{ \pm}}$ & 44 & 103 & 183 & 266 \\
\hline$m_{\tilde{\chi}_{2}^{ \pm}}$ & 620 & 876 & 945 & 1060 \\
\hline$m_{\tilde{t}_{1}}$ & 1068 & 805 & 1250 & 1390 \\
\hline$m_{\tilde{t}_{2}}$ & 1040 & 817 & 1260 & 1500 \\
\hline$m_{\tilde{c}_{1}}$ & & 2680 & 1740 & 1550 \\
\hline$m_{\tilde{c}_{2}}$ & & 2740 & 1740 & 1590 \\
\hline$m_{\tilde{u}_{1}}$ & & 2680 & 1740 & 1550 \\
\hline$m_{\tilde{u}_{2}}$ & & 2740 & 1740 & 1590 \\
\hline$m_{\tilde{b}_{1}}$ & & 781 & 1240 & 1240 \\
\hline$m_{\tilde{b}_{2}}$ & & 2460 & 1440 & 1500 \\
\hline$m_{\tilde{s}_{1}}$ & & 2680 & 1750 & 1430 \\
\hline$m_{\tilde{s}_{2}}$ & & 2460 & 1440 & 1590 \\
\hline$m_{\tilde{d}_{1}}$ & & 2680 & 1750 & 1430 \\
\hline$m_{\tilde{d}_{2}}$ & & 2460 & 1440 & 1590 \\
\hline$m_{\tilde{\tau}_{1}}$ & & 2460 & 975 & 88 \\
\hline$m_{\tilde{\tau}_{2}}$ & & 2510 & 1220 & 525 \\
\hline$m_{\tilde{\mu}_{1}}$ & & 2470 & 975 & 111 \\
\hline$m_{\tilde{\mu}_{2}}$ & & 2510 & 1220 & 522 \\
\hline$m_{\tilde{e}_{1}}$ & & 2460 & 975 & 111 \\
\hline$m_{\tilde{e}_{2}}$ & & 2510 & 1220 & 522 \\
\hline
\end{tabular}

TABLE III. The mass and mixing angle of the additional neutral gauge boson, tree level Higgs masses, neutralinos, charginos, and physical sparticle masses (in $\mathrm{GeV}$ ). Note that the spectrum of the first example (a toy model) is not realistic. 